\title{
Mobilidade funcional em indivíduos com paralisia cerebral espástica de acordo com o tipo e a idade
}

\author{
Ana F.R. Kleiner ${ }^{1}$ \\ Thiago G. Ayres ${ }^{1}$ \\ Patrícia M. Saraiva ${ }^{1}$ \\ Rosangela A. Batistela ${ }^{1}$ \\ Ricardo S. Pietrobon ${ }^{2}$ \\ Lilian T.B. Gobbi ${ }^{1}$
}

https://doi.org/10.5628/rpcd.08.03.406

\author{
${ }^{1}$ Universidade Estadual Paulista \\ Instituto de Biociências \\ Departamento de Educação Física \\ Laboratório de Estudos da Postura e da Locomoção \\ Rio Claro \\ Brasil \\ ${ }^{2}$ Duke University \\ Medical Center \\ Center of Excelence in Surgicals Outcomes \\ Durhan \\ USA
}

\section{RESUMO}

Mobilidade funcional é a habilidade de se locomover independentemente no ambiente, atingindo o destino desejado.

Indivíduos com paralisia cerebral espástica apresentam dificuldades na mobilidade de acordo com o tipo (diplegia, hemiplegia e tetraplegia). Além disso, com o avanço da idade, indivíduos portadores de paralisia cerebral espástica apresentam deterioração nos parâmetros cinemáticos da marcha. Assim, o objectivo deste estudo foi comparar a mobilidade funcional entre indivíduos com paralisia cerebral espástica, quanto ao tipo e ao grupo etário. Participaram deste estudo 68 portadores de paralisia cerebral espástica, sendo 30 diplégicos, 23 hemiplégicos e 15 tetraplégicos. Estes também foram distribuídos em 3 grupos etários: grupo crianças (4-12 anos); grupo jovens (13-19 anos) e grupo adultos (20-45 anos). A mobilidade funcional foi avaliada pela Escala de Mobilidade Funcional. O tempo gasto e a classificação na escala foram tratados por meio de ANOVAs não-paramétricas, separadamente para o tipo e o grupo etário, em 3 distâncias: $5 \mathrm{~m}, 50 \mathrm{~m}$ e $500 \mathrm{~m}$. Os resultados não evidenciaram diferenças quanto ao grupo etário e apontaram que indivíduos com paralisia cerebral espástica hemiplégica obtiveram melhores resultados para todas as distâncias percorridas. Podemos concluir que o comprometimento segmentar e o repertório motor afectam a mobilidade funcional.

Palavras-chave: paralisia cerebral, mobilidade funcional, grupo etário, espasticidade

\section{ABSTRACT \\ Functional mobility in spastic cerebral palsy individuals according to the type and age}

Functional mobility refers to the ability to move independently in the environment. Spastic cerebral palsy individuals show mobility needs according with the type (diplegia, hemiplegia and tetraplegia). With the age advance, spastic cerebral palsy individuals show deterioration on gait kinematics' parameters. The purpose of this study was to compare functional mobility among spastic cerebral palsy individuals according to the type and the age. Sixty eight individuals with spastic cerebral palsy, 30 with diplegia, 23 with hemiplegia and 15 with tetraplegia, participated in this study. They were also distributed in 3 age groups: children group (from 4 to 12 years old); young group (from 13 to 19 years old) and adult group (from 20 to 45 years old). The functional mobility was assessed by the Functional Mobility Scale. The spent time and the scale classification were treated by non-parametric ANOVA, by type and age group, in three distances: $5 \mathrm{~m}, 50 \mathrm{~m}$, and $500 \mathrm{~m}$. The results did not reveal age group differences and they showed that spastic cerebral palsy individuals with hemiplegia had better results for all distances. The segmental involvement and motor repertoire affect the functional mobility.

Key-words: cerebral palsy, functional mobility, age groups, spasticity 


\section{INTRODUÇÃO}

A espasticidade, definida como uma resistência velocidade-dependente do músculo ao alongamento passivo(13,17), ocorre após lesão no neurônio motor superior, que diminui o sinal cortical às vias descendentes. A perda dos sinais descendentes inibitórios ${ }^{(4,}$ 23, 32) afecta os reflexos relacionados à contracção muscular(13,17), embora as estruturas envolvidas ainda não sejam totalmente conhecidas $(23,32)$. Na paralisia cerebral espástica (PCE), muitos factores interagem para produzir limitações na execução dos movimentos, tais como: fraqueza muscular, restrições no comprimento muscular, pobre controle motor selectivo e sinais positivos de espasticidade ${ }^{(11}$, 18, 32). Como consequência, estas limitações geram dificuldades de mobilidade.

A mobilidade funcional (MF) pode ser definida como o meio pelo qual um indivíduo se move no ambiente para obter uma interacção diária com a família e a sociedade(15). É a habilidade do indivíduo mover o seu corpo de forma competente e independente, durante as tarefas do dia-a-dia(29).

Baseados na Classificação Internacional de Funcionalidade, Incapacidade e Saúde (ICF) da Organização Mundial da Saúde(31), Graham et al.(15) criaram a Escala de Mobilidade Funcional (EMF) para observar a função locomotora em ambientes quotidianos de crianças portadoras de Paralisia Cerebral (PC). A EMF classifica em 6 níveis a MF de acordo com o tipo de equipamento utilizado incluindo a marcha independente. É uma ferramenta simples e de fácil aplicação, onde os pesquisadores perguntam aos pais ou responsáveis a forma como o indivíduo se locomove em sua residência, escola e comunidade, respectivamente representados pelas distâncias de $5 \mathrm{~m}, 50 \mathrm{~m}$ e $500 \mathrm{~m}^{(15)}$.

Para validação da EMF, Graham et al.(15) examinaram uma amostra de 310 crianças com PCE e compararam a EMF com escalas já existentes ("Rancho Scale", "Child Health Questionnarie - Australian CHQ PF-50", e "Pediatric Outcomes Data Collection Instrument - versão 25") e instrumentos de medida de função física ("Uptimer" e Gasto Energético). O teste de Spearman revelou correlação alta e moderada com todas as ferramentas de medida, excepto com o teste de Gasto Energético. As maiores correlações $(p<0,001)$ foram observadas para o "Pediatric Outcomes Data Collection
Instrument - versão 25 ” (0,89 para os $5 \mathrm{~m}, 0,84$ para os $50 \mathrm{~m}$ e 0,82 para os $500 \mathrm{~m})$ e para o "Uptimer" $(0,87$ para os $5 \mathrm{~m}, 0,83$ para os $50 \mathrm{~m}$ e 0,84 para os $500 \mathrm{~m}$ ). Desde então, a EMF tem sido amplamente utilizada para mensurar as habilidades locomotoras, antes e após procedimento cirúrgico, de crianças com $\mathrm{PC}(15$, $19,25,26,28)$ e com poliomielite ${ }^{(12)}$ e tem-se mostrado sensível a mudanças após intervenção cirúrgica e ao método cirúrgico empregue $(19,25,26)$. Por outro lado, Thomason et al.(28) não observaram diferenças significativas na EMF entre programas de treinamento de força e de fisioterapia regular em nenhuma das distâncias propostas.

Os tipos de PCE são seleccionados em função das manifestações clínicas da espasticidade, que dependem da extensão das lesões neurológicas e do tipo de dano no SNC, do local da lesão e da capacidade do SNC em se adaptar e se reorganizar depois da lesão (plasticidade neural) ${ }^{(16,17)}$. Vários estudos aplicam a EMF em diplégicos, hemiplégicos e tetraplégicos ${ }^{(15}$, 24), apenas em diplégicos $(25,26,28)$ e em diplégicos e tetraplégicos ${ }^{(19)}$. Nenhuma destas pesquisas comparou a classificação na EMF entre os tipos de PCE. Para a classificação na EMF, todos os estudos estão embasados na opinião dos pais ou responsáveis sobre as habilidades locomotoras das crianças. Além disso, nenhum desses estudos observou a faixa etária dos pacientes.

O presente estudo foi planejado para aprofundar a questão do grupo etário por meio da inclusão de um grupo de adultos. Apesar da PC ser uma lesão não progressiva no SNC, as deficiências e incapacidades podem mudar ao longo do tempo(6, 7,13$)$. Assim, espera-se que os processos de crescimento e desenvolvimento e as compensações estruturais ósseas, articulares e musculares possam vir a influenciar a MF. Neste contexto, o objectivo deste estudo foi comparar a MF entre portadores de PCE agrupados por tipo (diplégica, hemiplégica e tetraplégica) e por idade (crianças, jovens e adultos), independente de género.

\section{MATERIAL E MÉTODO}

\section{Amostra}

Participaram deste estudo 68 portadores de PCE, sendo 30 com PCE diplégica (PCED), 23 com PCE hemiplégica (PCEH) e 15 com PCE tetraplégica (PCET). Os indivíduos foram distribuídos em 3 gru- 
Quadro 1. Distribuição dos participantes por grupo, quanto ao tipo de PCE, ao género e à idade.

\begin{tabular}{lccccccc}
\hline Grupo & $\mathrm{n}$ & \multicolumn{3}{c}{ Tipo de PCE } & & Gênero & Idade \\
\hline GC & & PCED & PCEH & PCET & M & F & \\
\hline GJ & 24 & 10 & 10 & 4 & 5 & 19 & $7,96(2,31)$ \\
\hline GA & 19 & 11 & 3 & 5 & 8 & 11 & $15,26(2,17)$ \\
\hline Total & 25 & 9 & 10 & 6 & 7 & 18 & $29,29(6,9)$ \\
\hline
\end{tabular}

n: número de participantes; GC: grupo criança; GJ: grupo jovem; GA: grupo adulto; PCED: paralisia cerebral espástica diplégica; PCEH: paralisia cerebral espástica hemiplégica; PCET: paralisia cerebral espástica tetraplégica; M: género masculino; F: género feminino.

pos etários: grupo crianças (GC: 4-12 anos; 24 participantes), grupo jovens (GJ: 13-19 anos; 19 participantes) e grupo adultos (GA: 20-45 anos; 25 participantes), correspondendo às fases da infância, adolescência e idade adulta (14). Como critério de inclusão no estudo, os voluntários deveriam apresentar mobilidade independente e, preferencialmente, nenhuma redução da capacidade intelectual, conforme avaliação realizada nas instituições de origem.

Os participantes deste estudo foram seleccionados nas Associações de Pais e Amigos dos Excepcionais (APAEs) e Centros de Reabilitação das cidades de Rio Claro/SP e região. Os pais ou responsáveis assinaram o termo de consentimento livre e esclarecido, autorizando a participação no estudo, e todo o procedimento experimental seguiu a Declaração de Helsínquia. O Quadro 1 apresenta as características de cada grupo.

\section{Procedimentos}

Para a obtenção dos dados da EMF cada participante, individualmente, foi convidado a percorrer, da maneira que mais lhe convinha, 3 distâncias $(5 \mathrm{~m}$, $50 \mathrm{~m}$ e $500 \mathrm{~m}$ ) em um trajecto previamente determinado em sua instituição. Uma trena com precisão em milímetros foi utilizada para delimitar os 3 trajectos percorridos.

Durante a colecta de dados, intervalos entre as distâncias foram fornecidos de acordo com a necessidade de cada participante. Da mesma forma, sempre que solicitadas pelo participante durante qualquer percurso, pausas para descanso, que não envolviam sentar, foram concedidas. Durante todos os trajectos, um avaliador permaneceu próximo ao participante.

\section{Escala de Mobilidade Funcional(15)}

Avalia a forma de locomoção de portadores de PC nas distâncias de 5m,50m e 500m. A EMF classifica as habilidades de locomoção em:

1 - Utiliza cadeira de rodas, cadeira motorizada ou carrinho: pode ficar em pé e pode andar apoiado por outra pessoa ou utilizando um andador/cadeira de roda, motorizada, etc.;

2 - Utiliza andador: sem a ajuda de outra pessoa; 3 - Utiliza duas muletas: sem ajuda de outra pessoa; 4 - Utiliza uma muleta ou duas bengalas: sem ajuda de uma outra pessoa;

5 - Independente do nível da superfície: Não usa nenhum acessório para andar ou precisa de alguma ajuda de outra pessoa. Se usa como suporte algum móvel, paredes, alambrados, frente de lojas, utilize o item 4 como a descrição mais apropriada; 6 - Independente em todas as superfícies: não usa nenhum acessório para andar ou precisa de alguma ajuda de outra pessoa quando anda, corre, sobe degraus e escadas.

\section{Variáveis dependentes}

As variáveis, mensuradas para cada uma das três distâncias percorridas pelos participantes, foram: a classificação na EMF e o tempo gasto (em segundos). Contrariamente aos estudos que classificaram a MF por meio da opinião dos pais $(15,19,25,26,28)$, o presente estudo solicitou que o voluntário executasse cada uma das distâncias previstas podendo, assim, aplicar tanto a classificação da EMF como o tempo gasto para percorrê-la. Além disso, o tempo gasto foi mensurado em estudo piloto(2) e revelou diferenças significativas entre as distâncias. 


\section{Colecta dos Dados}

Para aumentar a confiabilidade dos dados, observadores foram treinados e familiarizados com a EMF. No momento em que o participante percorreu qualquer uma das três distâncias, três observadores estavam estrategicamente posicionados para avaliá-lo: o primeiro acompanhando-o, e os outros posicionados no início e no final do trajecto. Os avaliadores codificaram o desempenho de acordo com a classificação da EMF. O tempo gasto foi mensurado com o auxílio de um cronómetro digital da marca SONY com precisão de centésimos de segundo, que foi accionado pelo primeiro observador.

\section{Análise Estatística}

Inicialmente, os dados foram tratados por meio de estatística descritiva (médias e desvios padrão). O teste de normalidade de Shapiro-Wilks revelou que as variáveis não se comportavam na curva normal. Assim, os dados foram tratados por meio de duas análises de variância não-paramétricas de KruskalWallis: a primeira tendo o tipo de PCE como fator e a segunda o grupo etário. Onde as ANOVAs apontaram diferenças significativas $(p \leq 0,05)$, o teste $U$ de Mann-Whitney foi empregue para localizá-las $(p \leq 0,01)$.

\section{RESULTADOS}

O Quadro 2 apresenta o número de participantes por distância percorrida, por tipo de PCE e por grupo etário. Pode observar-se que à medida que a distância percorrida aumenta o número de participantes que completa o percurso diminui. Assim, não há distribuição homogénea dos participantes quanto ao tipo de PCE e às distâncias. Em relação aos grupos etários, o Quadro 2 também revela que o GC apresentou o menor número de indivíduos completando as longas distâncias (50 e 500m). Os grupos GJ e GA apresentaram diminuição de participantes para a realização dos $500 \mathrm{~m}$.

Contudo, há necessidade de identificar o número de participantes de acordo com a classificação na EMF por tipo de PCE e por grupo etário (Quadro 3). Em termos gerais, a maior concentração de participantes ocorreu nas classificações 5 e 6 da EMF, independente da distância, do grupo etário e do tipo de PCE. Quanto à classificação da EMF, a análise de variância
Quadro 2. Número de participantes por distância percorrida, por tipo de $P C E$ e por grupo etário.

\begin{tabular}{lccc}
\hline Variáveis Independentes & \multicolumn{3}{c}{ Distâncias EMF } \\
\hline PCED & $\mathbf{5 m}$ & $\mathbf{5 0 ~ m}$ & $\mathbf{5 0 0 ~ \mathrm { m }}$ \\
\hline PCEH & 30 & 30 & 19 \\
\hline PCET & 23 & 21 & 20 \\
\hline GC & 15 & 11 & 10 \\
\hline GJ & 24 & 20 & 13 \\
\hline GA & 19 & 19 & 15 \\
\hline
\end{tabular}

PCED: paralisia cerebral espástica diplégica; PCEH: paralisia cerebral espástica hemiplégica; PCET: paralisia cerebral espástica tetraplégica; GC: grupo criança: GJ: grupo jovem; GA: grupo adulto.

Quadro 3. Número de participantes de acordo com a classificação da $E M F$ por grupo etário e para o tipo de $P C E$.

\begin{tabular}{|c|c|c|c|c|c|c|c|}
\hline \multirow[t]{2}{*}{ Distância } & \multirow[t]{2}{*}{ EMF } & \multicolumn{3}{|c|}{ Grupo Etário } & \multicolumn{3}{|c|}{ Tipo } \\
\hline & & GC & GJ & GA & PCED & PCEH & PCET \\
\hline \multirow[t]{6}{*}{$5 \mathrm{~m}$} & 1 & 6 & 5 & 2 & 10 & 1 & 2 \\
\hline & 2 & 2 & - & 4 & 4 & - & 2 \\
\hline & 3 & - & 1 & 2 & 3 & - & - \\
\hline & 4 & 1 & 1 & 1 & - & - & 3 \\
\hline & 5 & 5 & 4 & 8 & 3 & 8 & 5 \\
\hline & 6 & 10 & 8 & 8 & 10 & 14 & 3 \\
\hline \multirow[t]{6}{*}{$50 \mathrm{~m}$} & 1 & 4 & 5 & 2 & 10 & - & 1 \\
\hline & 2 & 2 & - & 3 & 4 & - & 1 \\
\hline & 3 & - & 1 & 2 & 3 & - & - \\
\hline & 4 & 1 & 1 & - & - & 1 & 1 \\
\hline & 5 & 5 & 4 & 9 & 4 & 8 & 6 \\
\hline & 6 & 8 & 8 & 7 & 9 & 12 & 2 \\
\hline \multirow[t]{6}{*}{$500 \mathrm{~m}$} & 1 & 2 & 2 & 1 & 4 & - & 1 \\
\hline & 2 & - & - & 3 & 2 & - & 1 \\
\hline & 3 & - & - & 2 & 2 & - & - \\
\hline & 4 & 4 & 3 & 1 & 5 & 1 & 2 \\
\hline & 5 & 3 & 4 & 6 & - & 9 & 4 \\
\hline & 6 & 4 & 6 & 8 & 6 & 10 & 2 \\
\hline
\end{tabular}

EMF: escala de mobilidade funcional; GC: grupo criança: GJ: grupo jovem; GA: grupo adulto; PCED: paralisia cerebral espástica diplégica; PCEH: paralisia cerebral espástica hemiplégica; PCET: paralisia cerebral espástica tetraplégica. 

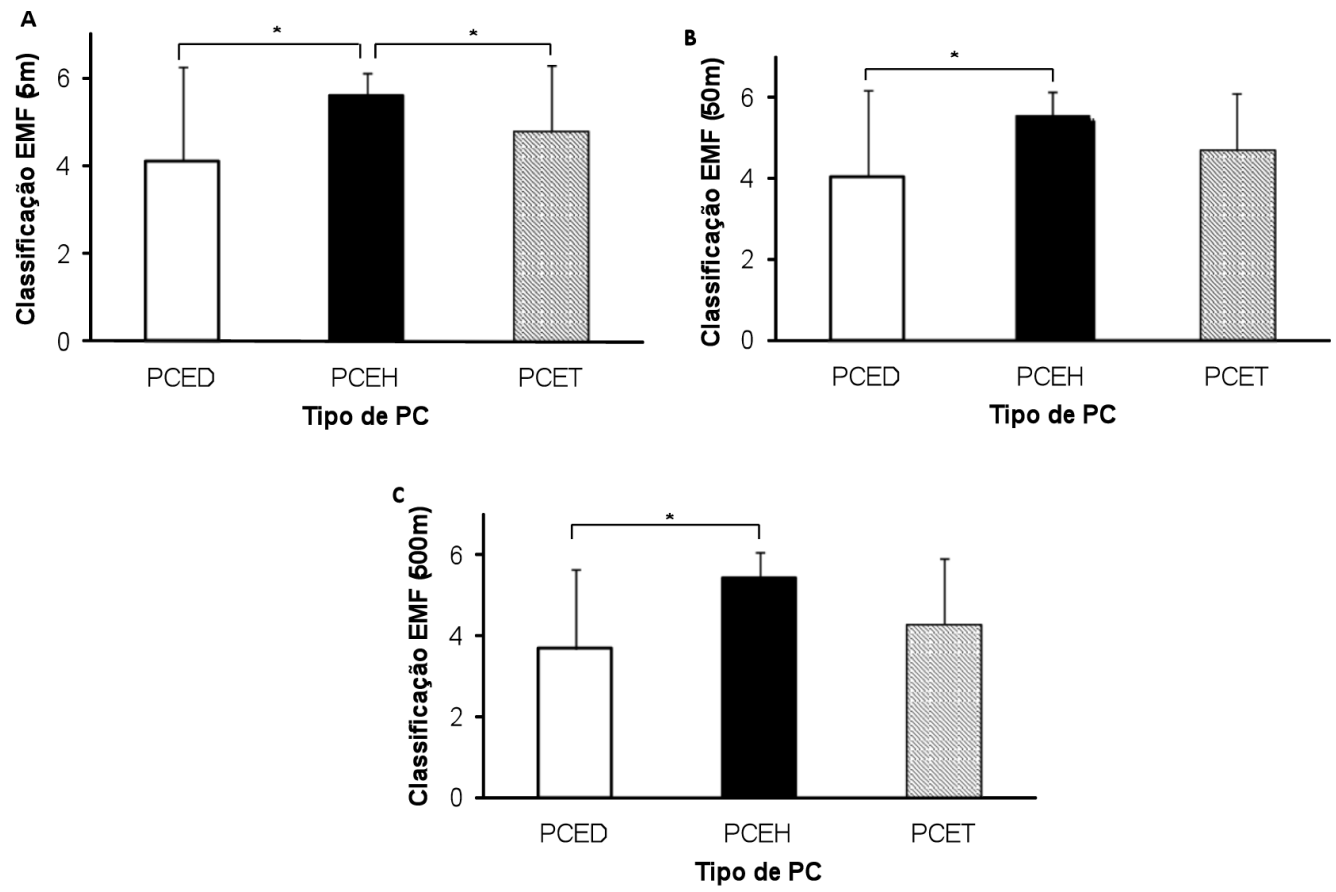

Figura 1. Médias e desvios padrão das classificações da $\varepsilon M F$ para o tipo de $P C E$ : $A=5 m, B=50 m$ e $C=500 m$. EMF: $\varepsilon s c a l a$ de Mobilidade Funcional; PCED: paralisia cerebral espástica diplégica; PCEH: paralisia cerebral espástica hemiplégica; PCET: paralisia cerebral espástica tetraplégica. [* diferença significativa entre os tipos de PCE]
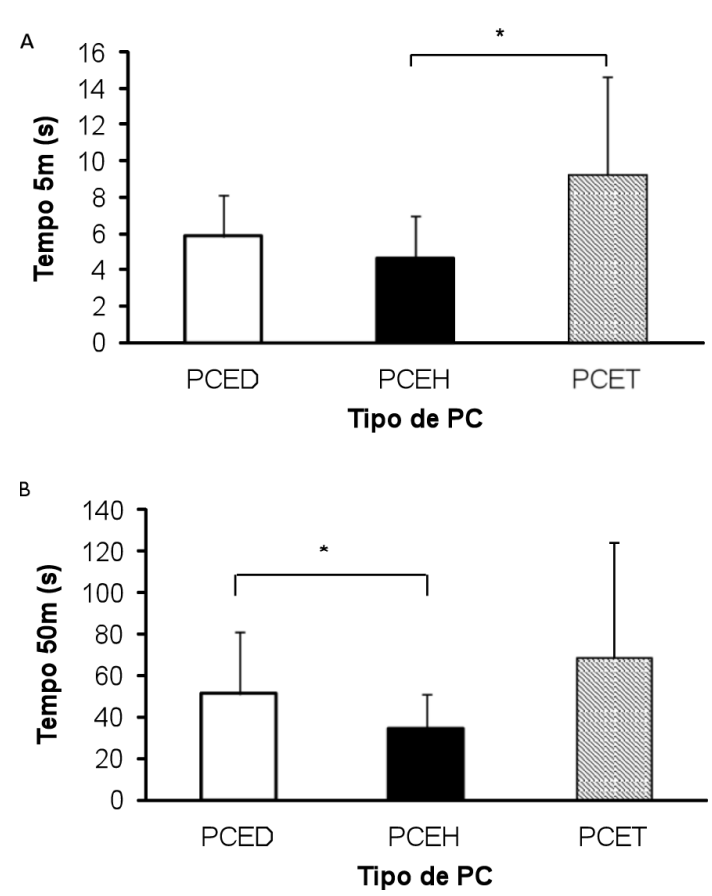

Figura 2. Médias e desvios padrão do tempo gasto por tipo de $P C E$ : $A=5 m$ e $B=50 m$. PCED: paralisia cerebral espástica diplégica; $P C E H$ : paralisia cerebral espástica hemiplégica; PCET: paralisia cerebral espástica tetraplégica. ( diferença significativa entre os tipos de $P C E$ ]

não paramétrica de Kruskall-Wallis revelou efeito principal de tipo de PCE para os $5 \mathrm{~m}(\mathrm{H}=11,683$; $\mathrm{p} \leq 0,003$; Figura 1A). O teste não paramétrico de Mann-Whitney localizou as diferenças entre os tipos de PCED e PCEH ( $U=187,00 ; p \leq 0,003)$ e PCEH e PCET $(U=79,00 ; p \leq 0,004)$. Para os $50 \mathrm{~m}$, a análise de Kruskall-Wallis também evidenciou efeito principal de tipo de PCE na classificação da EMF $(\mathrm{H}=$ 11,693; p s 0,003; Figura 1B). Diferenças apenas entre os tipos de PCED e PCEH foram localizadas pelo teste de Mann-Whitney ( $U=155,00$; $\mathrm{p} \leq 0,001$ ). Para a classificação da EMF nos 500m, a análise de Kruskall-Wallis demonstrou efeito principal de tipo de PCE $(H=9,643 ; p \leq 0,008$; Figura 1C). Foram localizadas apenas diferenças entre os 
tipos de PCED e PCDH pelo teste de Mann-Whitney nessa distância $(U=92,50 ; p \leq 0,005)$.

Quanto ao tempo gasto para realizar as tarefas, a análise de Kruskall-Wallis evidenciou efeito principal de tipo de PCE no tempo dos $5 \mathrm{~m}(\mathrm{H}=9,682$; $\mathrm{p} \leq 0,008$; Figura 2A). O teste de Mann-Whitney localizou diferença apenas entre PCEH e PCET ( $\mathrm{U}=$ 81,00; p $\leq$ 0,006). Para a distância de 50m, a análise de Kruskall-Wallis mostrou efeito principal de tipo de PCE $(H=8,046 ; p \leq 0,018$; Figura 2B). Diferença apenas entre os tipos de PCED e PCEH foi evidenciada pelo teste de Mann-Whitney ( $\mathrm{U}=170,00$; $\mathrm{p} \leq$ 0,006). A análise de Kruskall-Wallis não evidenciou efeito principal de tipo de PCE no tempo dos $500 \mathrm{~m}$ $(\mathrm{H}=4,049 ; \mathrm{p} \leq 0,132)$.

A ANOVA não evidenciou efeito principal de grupo etário para as variáveis tempo gasto para as distâncias de 5 , 50 e $500 \mathrm{~m}(\mathrm{H}=0,190 ; \mathrm{p}<0,909 ; \mathrm{H}=$ 1,194; $\mathrm{p}<0,550 ; \mathrm{H}=1,848$; $\mathrm{p}<0,397$; respectivamente), e classificação na EMF para as distâncias de 5, 50 e $500 \mathrm{~m}(\mathrm{H}=0,082 ; \mathrm{p}<0,960 ; \mathrm{H}=0,003$; $\mathrm{p}<0,999 ; \mathrm{U}=0,997 ; \mathrm{p}<0,607$; respectivamente).

\section{DISCUSSÃO}

O objectivo deste estudo foi comparar a MF entre portadores de PCE agrupados por tipo (hemiplégico, diplégico e tetraplégico) e por idade (crianças, jovens e adultos).

Caracterizando os resultados em relação ao tipo de PCE foi observado que quanto maior é o grau de comprometimento dos membros inferiores (diplégicos e tetraplégicos) menor o número de participantes que completa o percurso (Quadro 2). Os indivíduos PCEH apresentam maior concentração de mobilidade independente, de acordo com a classificação da EMF, quando comparados com os indivíduos PCED e PCET.

Em relação à classificação da EMF, a análise estatística evidenciou diferenças quanto ao tipo de PCE nas 3 distâncias (Figura 1). Para os 5m, os indivíduos com PCEH apresentaram as maiores classificações na EMF quando comparados aos grupos PCED e PCET. Para as distâncias mais longas (50 e 500m), o grupo PCED apresentou menor classificação na EMF quando comparado ao grupo PCEH. Cada tipo de PCE é decorrente de um tipo lesão e do período em que esta ocorreu. A PCEH, mais comum, está asso- ciada à lesão em um único hemisfério na maioria dos casos e suas causas mais frequentes são: alguns tipos de malformações cerebrais, acidentes vasculares ocorridos ainda na vida intra-uterina e traumatismos crânio-encefálicos. A PCED é associada à leucomalácia periventricular, onde as desordens do movimento ocorrem depois de hiperbilirrubinemia e lesão nos núcleos da base. A PCET está associada a lesões difusas graves no SNC (infecções, hipóxia e traumas) e a malformações cerebrais graves $(1,20,7)$. Desta forma, os indivíduos com PCEH possuem um dos membros inferiores não comprometido, ou seja, não afetado pela PC. A compensação do lado não afetado no desempenho da marcha tem sido amplamente documentada $(5,8,22)$.

Devido ao comprometimento grave nos indivíduos com PCET, esperava-se que este grupo apresentasse valores significativamente inferiores aos demais grupos em todas as distâncias. Todavia, valores intermediários foram observados em todas as distâncias, sendo significativos apenas nos $5 \mathrm{~m}$. Uma possível explicação para este resultado é que há uma alteração na distribuição dos indivíduos com PCET na classificação da EMF, sendo que houve concentração no nível 5 para aqueles que completaram a tarefa. Neste nível, o participante apresenta a locomoção independente, mas não possui um repertório motor para variar as formas de locomoção.

Também foram encontradas diferenças significativas em relação ao tempo gasto nos $5 \mathrm{~m}$ e nos $50 \mathrm{~m}$ quanto ao tipo de PCE (Figura 2). Para os $5 \mathrm{~m}$, foram encontradas diferenças entre PCEH e PCET e para os $50 \mathrm{~m}$ entre PCEH e PCED. Estes resultados indicam que quanto maior o comprometimento nos membros inferiores, menores são as classificações na EMF e maiores são os tempos para percorrer as distâncias, especificamente os $5 \mathrm{~m}$. Adaptações locomotoras funcionais, como o uso de equipamentos, são utilizadas por indivíduos que possuem os dois membros inferiores acometidos pela $\mathrm{PC}$ e, consequentemente, maior tempo é gasto na locomoção. A função motora de portadores de PC é influenciada primariamente pelos sinais negativos, especialmente a fraqueza muscular. O baixo nível de actividade física, a diminuição dos impulsos motores, as mudanças nas propriedades elásticas dos músculos, as alterações nas vias de inibição recíproca dos músculos agonistas e antagonis- 
tas e a espasticidade podem contribuir para a fraqueza muscular(9). Além disso, a força no membro inferior interfere na velocidade do andar(10).

Não foram encontradas diferenças estatisticamente significativas na classificação da EMF e no tempo gasto para os grupos etários. O grupo criança evidenciou maior número de desistências para as grandes distâncias, quando comparado aos grupos jovem e adulto. Em observação directa durante a colecta dos dados, os indivíduos do grupo criança demonstraram cansaço e desistiram de completar as distâncias mais longas. Ainda, os três grupos etários, em relação à classificação da EMF, apresentaram maior concentração de indivíduos com mobilidade independente (níveis 5 e 6 da EMF; Quadro 3). Pirpiris e Graham (24) também não encontraram diferenças no teste de Uptimer entre grupos etários (crianças e adolescentes, entre 8 e 14 anos). Contudo, em relação à locomoção de indivíduos com PCE, estudos longitudinais têm revelado que há deterioração nos parâmetros cinemáticos da marcha com o avanço da idade $(16,21)$, inclusive em adultos ${ }^{(6)}$.

Duas possíveis explicações podem ser aplicadas nestes resultados: uma estrutural e outra relacionada à experiência motora. Em relação à explicação estrutural, Johnson et al.(16), com crianças PCED, revelaram que existe um relacionamento entre o crescimento ósseo e as mudanças desenvolvimentais observadas na marcha, indicando diminuição funcional com o passar do tempo. Wright e Rang(30), utilizando um modelo de rato espástico, relataram que a espasticidade pode causar contraturas musculares e deformidades articulares, em função do crescimento muscular não acompanhar o crescimento ósseo. Os resultados do presente estudo não confirmam os resultados de Johnson et al.(16) e Wright e Rang(30).

Para a segunda explicação, Thelen(27) pontua que, no curso do desenvolvimento, novos comportamentos são decorrentes da resolução de problemas da nova tarefa (desafio motor), utilizando o princípio de exploração e selecção. Em complementação, o repertório motor do indivíduo aumenta à medida que novas situações são exploradas e componentes adequados são seleccionados(3). Desta forma, é razoável supor que indivíduos com maior idade cronológica tenham enfrentado maior número de desafios motores e, portanto, possuam maior repertório motor. A experiência em tarefas locomotoras e o atendimento em centros de reabilitação poderiam, assim, diminuir os efeitos estruturais da idade e permitir que jovens e adultos tivessem melhor desempenho que as crianças. Os resultados deste estudo confirmam o princípio de exploração e seleção proposto por Thelen(27), pois o número de adultos e de jovens que completou a distância mais longa, nos níveis de locomoção independente, foi maior que o de crianças.

Em relação à classificação na EMF e ao tempo gasto para percorrer as distâncias, não houve melhor desempenho dos adultos. Bottos et al.(6) observaram que muitos pacientes, ao atingir a idade adulta, não foram capazes de manter a locomoção independente ou com o uso de equipamentos.

\section{CONCLUSÃO}

Os resultados do presente estudo permitem concluir que: (i) a proporção de indivíduos que completou as 3 distâncias propostas é menor no grupo de crianças; (ii) em relação à classificação na MF, os indivíduos PCEH apresentaram maiores valores que os demais tipos de PCE para todas as distâncias percorridas, pela compensação do lado não comprometido; (iii) o grupo etário não altera a classificação na EMF; (iv) o maior repertório motor de jovens e adultos proporcionou que a maioria destes indivíduos percorresse as distâncias mais longas.

\section{AGRADECIMENTOS}

Os autores agradecem o suporte financeiro da FINEP.

\section{CORRESPONDÊNCIA}

\section{Lilian Teresa Bucken Gobbi}

Avenida 25 A, 1515

13.506-900 Rio Claro São Paulo Brasil

telefone/fax: +55 19 3534-6436

E-mail: ltbgobbi@rc.unesp.br 


\section{REFERÊNCIAS}

1. Arcadi J, Bax M (1998). Cerebral Palsy. In: Arcadi J (ed.). Diseases of motor system in childhood. 2ed. London: Mac Keith, 210-39

2. Ayres TG, Kleiner AFR, Saraiva PM, Pietrobon, RS, Gobbi, LTB (2005). Análise da mobilidade funcional de indivíduos com paralisia cerebral espástica de acordo com a idade. Revista da SOBAMA (Sociedade Brasileira de Atividade Motora Adaptada) 10, 1:51-2

3. Barela JA (2001). Ciclo percepção-ação no desenvolvimento motor. In: Teixeira LA (ed.). Avanços em Comportamentos Motor. Rio Claro: Movimento, 40-61

4. Barnes MP (2003). Spasticity in multiple sclerosis. Neurorehab Neural Re 17, 1:66-70

5. Berger W, Quintern J, Dietz V (1982). Pathophysiology of gait in children with cerebral palsy. Electroencephalogr Clin Neurophysiol 53, 5:538-48

6. Bottos M, Feliciangeli A, Sciuto L, Gericke C, Vianello A (2001), Functional status of adults with cerebral palsy and implications for treatment of children. Dev Med Child Neurol 43:516-28

7. Dabney KW, Lipton GE, Miller F (1997). Cerebral Palsy. Curr Opin Pediatr 9:81-8

8. Damiano DL, Abel MF (1998). Functional outcomes of strength training in spastic cerebral palsy. Arch Phys Med Rehabil 79:119-25

9. Damiano DL, Dodd K, Taylor NF (2002). Should we be testing and training muscle strength in cerebral palsy? Dev Med Child Neurol 44:68-72

10. Damiano DL, Kelly LE, Vaughan CL (1995). Effects of a quadriceps femoris strengthening program on crouch gait in children with cerebral palsy. Phys Ther 75:658-67

11. Damiano DL, Laws E, Carmines DV, Abel MF (2006). Relationship of spasticity to knee angular velocity and motion during gait in cerebral palsy. Gait Posture 23:1-8

12. Emara KM, Khames A (2007). Functional outcome after lengthening with and wothout deformity correction in pólio patients. Int Orthop (SICOT) http://www.springerlink.com/content/2515627k4px30m44/fulltext.pdf

13. Flett PJ (2003). Rehabilitation of spasticity and related problems in childhood cerebral palsy. J Paediatr Child Health 29:6-14

14. Freiberg KL (1987). Human development: a life-span approach. 3 ed. Boston, Jones and Bartlett.

15. Graham HK, Harvey A, Rodda J, Nattrass GR, Pirpiris M (2004). The functional mobility scale (FMS). J Pediatr Orthop 25, 5:514-20

16. Johnson DC, Damiano DL, Abel MF (1997). The evolution of gait in childhood and adolescent cerebral palsy. J Pediatr Orthop 17:392-6

17. Koman LA, Smith BP, Shilt JS (2004). Cerebral Palsy. Lancet 363:1619-31

18. Latach ML, Penn RD (1996). Changes in voluntary motor control induced by intrathecal bacoflen in patients with spasticty of different etiology. Physiother Res Int 1:229-46

19. Ma FYP, Selber P, Nattrass GR, Harvey AR, Wolf R, Graham HK (2006). Lengthening and transfer of hamstrings for a flexion deformity of the knee in children with bilateral cerebral palsy. J Bone Joint Surg Br 88, 2:248-54

20. Nelson KB, Swaiman KF, Russman SR (1994). Cerebral Palsy. In: Swaiman KF (ed.). Pediatric neurology: principles and practice. St. Louis: Mosby Company, 471-88

21. Norlin R, Odenrick P (1986). Development of gait in spastic children with cerebral palsy. J Pediatr Orthop 6:674-80

22. O'Byrne JM, Jenkinson A, O'Brien TM (1988). Quantitative analysis and classification of gait patterns in cerebral palsy using a three-dimensional motion analyzer. $J$ Child Neurol 13, 3:101-8

23. Penn RD, Corcos DM (1990). Spasticity and its Management. In: Youngmans JR (ed.). Neurological Surgery $3^{\text {rd }}$ Ed. W. B. Saunders Co, 4371-85

24. Pirpiris M, Graham HK (2004). Uptime in children with cerebral palsy. J Pediatr Orthop 25, 5:521-8

25. Rodda JM, Graham HK, Nattrass MP, Galea MP, Baker R, Wolf R (2006). Correction of severe crouch gait in patients with spastic diplegia with use of multilevel. Orthopaedic Surgery. J Bone Joint Surg Am 88:2653-64

26. Rodda JM, Baker R, Galea M, Nattrass G, Graham HK (2006). The impact of single event multilevel surgery (semls) for severe crouch gait in spastic diplegic cerebral palsy: outcome at 5 years. Gait Posture 25S:S87-9

27. Thelen E (1995). Motor development: a new synthesis. Am Psychol 50, 2:79-95

28. Thomason P, Baker R, Dodd K, Taylor N, Graham K (2006). Pilot protocol for a randomized clinical trial: the effectiveness of single event multilevel surgery for children with cerebral palsy. Gait Posture 25S:S146-7

29. Williams EN, Carroll SG, Reddihough DS, Philips BA, Galea MP (2005). Investigation of timed "up \& go" test in children. Dev Med Child Neurol 47, 8:518-25

30. Wright J, Rang M (1989). The spastic mouse. Clin Orthop 253:12-9

31. World Health Organization (2001). International Classification of Functionning, Disability and Health - Short Version. Geneva: World Health Organization, 121-60 